\title{
Warm ionized ISM in the bulge of Andromeda galaxy
}

\author{
Marat Gilfanov ${ }^{1}$ and Akos Bogdan ${ }^{1}$ \\ ${ }^{1}$ Max-Planck-Institute for Astrophysics, Karl-Schwarzschild-Str. 1, D-85748 Garching \\ email: mgilfanov@mpa-garching.mpg.de
}

\begin{abstract}
We demonstrate that unresolved X-ray emission from the bulge of M31 is composed of at least three different components: (i) Broad-band emission from a large number of faint sources - mainly accreting white dwarfs and active binaries, associated with the old stellar population, similar to the Galactic Ridge X-ray emission of the Milky Way. (ii) Soft emission from ionized gas with temperature of about $\sim 300 \mathrm{eV}$ and mass of $\sim 4 \times 10^{6} \mathrm{M}_{\odot}$. The gas distribution is significantly elongated along the minor axis of the galaxy suggesting that it may be outflowing in the direction perpendicular to the galactic disk. The shadows cast on the gas by spiral arms and the 10-kpc star-forming ring confirm large off-plane extent of the gas. (iii) Hard unresolved emission from spiral arms, most likely associated with protostars and young stellar objects located in the star-forming regions.
\end{abstract}

